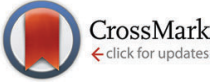

Cite this: Phys. Chem. Chem. Phys., $2016,18,13538$

Received 31st March 2016, Accepted 19th April 2016

DOI: $10.1039 / c 6 c p 02130 a$

www.rsc.org/pccp

\title{
The conformational space of the neurotransmitter serotonin: how the rotation of a hydroxyl group changes all $\dagger$
}

\author{
Martin Wilke, ${ }^{a}$ Christian Brand, ${ }^{\text {ab }}$ Josefin Wilke ${ }^{a}$ and Michael Schmitt*a
}

\begin{abstract}
The 5-hydroxytryptamine receptors $\left(5 \mathrm{HT}_{n}\right)$ are optimized for 5-hydrotryptamine molecules, resulting in a significantly enhanced psychoactive response compared with the 4-, 6-, 7-isomers. This is despite their relatively similar energetic stabilities, excited state lifetimes and emission characteristics. In this work we investigate the conformational space of serotonin (5-hydroxytryptamine) using a combination of rotationally resolved electronic spectroscopy and $a b$ initio calculations. The geometries of the four most abundant conformers are assigned from their molecular parameters in the electronic ground and excited state. We find a conformer-dependent competition between two polar groups trying to establish a hydrogen bond with the same $\mathrm{H}$-atom in the most stable conformer of serotonin. The result explains some remarkable deviations with respect to the conformational space of the closely related neurotransmitter tryptamine. Based on the comparison to other 5-substituted indoles we propose to generalize this finding to explain the conformational preferences of indole-based neurotransmitters.
\end{abstract}

\section{Introduction}

The neurotransmitter and hormone serotonin is a derivative of the aromatic amino acid tryptophan. It is the natural ligand which activates the 5-hydroxytryptamine (5-HT) receptors which are mostly $\mathrm{G}$ protein coupled receptors. ${ }^{1,2}$ The omnipresence of these receptors in the body leads to a multitude of different effects. ${ }^{3}$ Serotonin modulates neural activity, neuropsychological processes and has vascular effects (both vasoconstriction and vasodilation). Furthermore, it has cardiac functions and affects the respiratory system, the endocrine system and metabolism, as well as the gastrointestinal system. ${ }^{4}$ A deeper understanding of the interaction of neurotransmitters with their specific receptors requires a better knowledge of the influences which shape their conformational space.

A suitable nomenclature for the conformational space of serotonin is depicted in Fig. 1. ${ }^{5}$ For all experimentally observed conformers, the ethyl amino side chain is perpendicular to the aromatic plane which results in three possible conformations of the $\mathrm{NH}_{2}$ group: gauche to the pyrrole ring (Gpy), gauche position to the phenyl moiety (Gph) or pointing away from the

\footnotetext{
${ }^{a}$ Heinrich-Heine-Universität, Institut für Physikalische Chemie I, D-40225 Düsseldorf, Germany. E-mail: mschmitt@hhu.de; Fax: +49 211 8112179; Tel: +4902118112100

${ }^{b}$ Faculty of Physics, University of Vienna, VCQ, QuNaBioS, Boltzmanngasse 5, A-1090 Vienna, Austria

† Electronic supplementary information (ESI) available. See DOI: 10.1039/c6cp02130a
}

chromophore (Anti). For both gauche conformations the lone pair (LP) of the $\mathrm{NH}_{2}$ group can point up, out, or in. The in conformers, however, are much higher in energy and will be neglected in this study. For the Anti conformers the LP can point up or to either one of the rings: phenyl $(p h)$ or pyrrole $(p y)$. Finally, the orientation of the OH-group is denoted with anti when it is anti-parallel to the NH bond of the pyrrole ring, or syn- for the other option. In the end a set of 14 possible conformers exists which is shown in the online ESI. $\dagger$

An experimental investigation of the conformational space of the protonated form was performed by Lagutschenkov et al. ${ }^{6}$ Both the bare ${ }^{5}$ and the singly hydrated neutral serotonin ${ }^{7}$ were studied with vibrational resolution in the group of Zwier. They found eight conformers which they divided into a group of five transitions labeled as $A$ to $E$, blue-shifted by around $200 \mathrm{~cm}^{-1}$ from the second group of three transitions labeled as $F$ to $H .^{5}$ The lowest energy conformation was stated to be the Gpy(out)/ anti conformer like in the closely related neurotransmitter tryptamine which essentially is serotonin without the hydroxyl group. Later, this assignment was corrected by Cabezas et al. ${ }^{8}$ who established the Gph(out)/anti conformer to be the global minimum based on rotational spectroscopy. This is in agreement with theoretical predictions from Srivastava and Singh $^{9}$ who performed a comprehensive computational study on serotonin at various levels of theory. Also Van Mourik and Emson investigated the conformational space of serotonin and tryptamine theoretically. ${ }^{10}$

Apart from the conformational analysis the study of LeGreve et al. pointed to some intriguing irregularities in the vibronic 


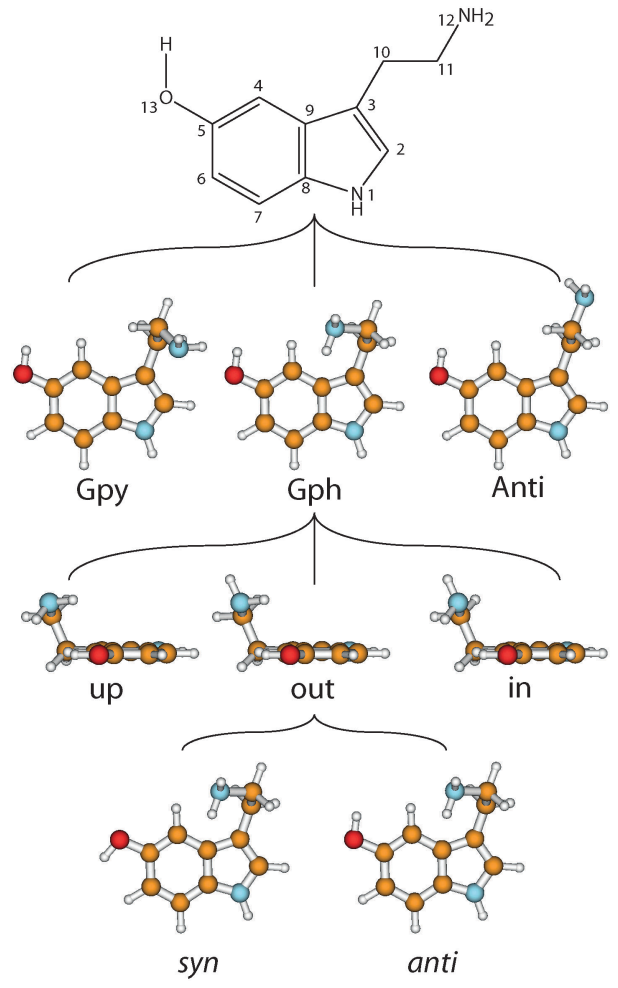

Fig. 1 Atomic numbering of the heavy atoms of serotonin and nomenclature of the conformers. The nomenclature is based on the orientation of the $\mathrm{NH}_{2}$ group, the orientation of its lone pair, and the two conformations of the $\mathrm{OH}$-group. For details see text.

spectrum of serotonin. ${ }^{5}$ The energetic gap between the synand the anti-conformer with respect to the hydroxyl group is relatively constant at $230 \mathrm{~cm}^{-1}$, close to the respective value for 5-hydroxindole. ${ }^{11,12}$ However, for the Gph(out) conformers the energetic gap is increased by $30 \mathrm{~cm}^{-1}$. This is very surprising as the only difference is a rotation about a single $\mathrm{OH}$-bond by $180^{\circ}$. Furthermore, the nitrogen atom of the ethyl amino side chain is $510 \mathrm{pm}$ away from the oxygen atom of this group, making a direct interaction unlikely. So far, this effect has been tentatively explained in the literature by electronic ${ }^{5,9}$ or dipoledipole interactions. ${ }^{6,7}$ Additionally, the most stable conformer of serotonin does not coincide with the energetically lowest one of tryptamine. Thus, the question arises which kind of mechanism leads to this remarkable stabilization and irregularity for the $\mathrm{Gph}$ (out)/anti conformer of serotonin.

In the present contribution, we use a combination of rotationally resolved electronic spectroscopy and high-level quantum mechanical calculations to investigate the conformational space of serotonin and compare it to that of tryptamine. Tryptamine itself has been studied at rotational ${ }^{13}$ and vibronic resolution ${ }^{14}$ as well as at partial ${ }^{15,16}$ and full ${ }^{17-19}$ rovibronic resolution. Seven different conformers were identified and assigned based on their rotational constants and vibrational spectra. Based on the comparison of the existing data on serotonin derivatives ${ }^{20-22}$ we propose the formation of intramolecular hydrogen bonds to be responsible for the conformational preference for many indole-based neurotransmitter. The results give new and important insights into the stabilization mechanisms of this complex class of molecules.

\section{Techniques}

\subsection{Experimental procedures}

Serotonin $(\geq 97 \%)$ was purchased from Wako and used without further purification. The experimental set-up for the rotationally resolved laser induced fluorescence spectroscopy is described in detail elsewhere. ${ }^{23}$ In brief, the laser system consists of a single frequency ring dye laser (Sirah Matisse DS) operated with Rhodamine 6G and Kiton Red, pumped with $8 \mathrm{~W}$ of the $514 \mathrm{~nm}$ line of an $\mathrm{Ar}^{+}$-ion laser (Coherent, Sabre 15 DBW). The dye laser output was coupled into an external folded ring cavity (Spectra Physics Wavetrain) for second harmonic generation. The resulting output power was constant at about $15 \mathrm{~mW}$ during the experiment. The molecular beam was formed by co-expanding serotonin, heated to $210{ }^{\circ} \mathrm{C}$, and $250 \mathrm{mbar}$ of argon through a $200 \mu \mathrm{m}$ nozzle into the vacuum chamber. The molecular beam machine consists of three differentially pumped vacuum chambers that are linearly connected by skimmers ( $1 \mathrm{~mm}$ and $3 \mathrm{~mm}$, respectively) in order to reduce the Doppler width. The resulting resolution is $18 \mathrm{MHz}$ (FWHM) in this set-up. In the third chamber, $360 \mathrm{~mm}$ downstream of the nozzle, the molecular beam crosses the laser beam at a right angle. The imaging optics set-up consists of a concave mirror and two plano-convex lenses to focus the resulting fluorescence onto a photomultiplier tube, which is mounted perpendicularly to the plane defined by the laser and molecular beam. The signal output was then discriminated and digitized by a photon counter and transmitted to a PC for data recording and processing. The relative frequency was determined with a quasi confocal Fabry-Perot interferometer. The absolute frequency was obtained by comparing the recorded spectrum to the tabulated lines in the iodine absorption spectrum. ${ }^{24}$

\section{Computational methods}

\subsection{Quantum chemical calculations}

Structure optimizations were performed employing Dunning's correlation consistent polarized valence triple zeta (cc-pVTZ) basis set from the Turbomole library. ${ }^{25,26}$ The equilibrium geometries of the electronic ground and the lowest excited singlet states were optimized using the approximate coupled cluster singles and doubles model (CC2) employing the resolution-ofthe-identity approximation (RI). ${ }^{27-29}$ Vibrational frequencies and zero-point corrections to the adiabatic excitation energies have been obtained from numerical second derivatives using the NumForce script ${ }^{30}$ implemented in the TuRbomole program suite. ${ }^{31}$ Second-order perturbation theory (MP2) optimizations were performed with the $6-311++\mathrm{G}(\mathrm{d}, \mathrm{p})$ basis set using the Gaussian 09 program package. ${ }^{32}$ For the MP2 optimized geometries a Natural Bond Orbital (NBO) analysis ${ }^{33}$ has been performed. 


\subsection{Fits of the rovibronic spectra using evolutionary algorithms}

The search algorithm employed for the fit of the rotationally resolved electronic spectra is an evolutionary strategy (ES) adapting normal mutations via a covariance matrix adaptation (CMA) mechanism. This (CMA-ES) algorithm was developed by Ostermeier and Hansen ${ }^{34,35}$ and is designed especially for optimization on rugged search landscapes that are additionally complicated due to noise, local minima and/or sharp bends. It belongs to a group of global optimizers that were inspired by natural evolution. For a detailed description of these evolutionary strategies refer to ref. 36-39.

\section{Results and discussion}

\subsection{Computational results}

The assignment of the experimental spectra and identification of the respective structures is based on high level $a b$ initio calculations. Table 1 shows the calculated molecular properties of the lowest 14 conformers of serotonin obtained from CC2/cc-pVTZ calculations. As the rotational constants are mainly determined by the orientation of the $\mathrm{NH}_{2}$ group, we can sort the conformers into three families (Gpy, Gph and Anti). However, inside a family the rotational constants are quite similar and an assignment solely based on their absolute values is difficult.

The orientation of the transition dipole moment (TDM) given by its angles $\theta$ with the inertial $a$-axis and $\phi$ with the $c$-axis (cf. Fig. 2), provides additional information to distinguish the conformers. The respective values for all 14 conformers are also listed in Table 1. While $\phi$ is quite similar for all conformers and no systematic differences can be observed, the changes of $\theta$ are a reliable measure to distinguish between syn- and anti-conformers:

Table 1 Calculated properties of the ground and lowest electronically excited state of the 14 lowest-energy conformers of serotonin at their respective CC2/cc-pVTZ optimized geometries. This includes the ground and excited state rotational constants. Double-primed constants belong to the ground state and single-primed to the excited state. The angle of the transition dipole moment with the main inertial a-axis is given by $\theta$ and with the $c$-axis by $\phi$. A positive sign of the angle corresponds to a clockwise rotation of the TDM vector onto the a-axis (cf. Fig. 2)

\begin{tabular}{lllllllll}
\hline & $A^{\prime \prime} /$ & $B^{\prime \prime} /$ & $C^{\prime \prime} /$ & $A^{\prime} /$ & $B^{\prime} /$ & $C^{\prime} /$ & & \\
Conformer & $\mathrm{MHz}$ & $\mathrm{MHz}$ & $\mathrm{MHz}$ & $\mathrm{MHz}$ & $\mathrm{MHz}$ & $\mathrm{MHz}$ & $\theta /^{\circ}$ & $\phi /^{\circ}$ \\
\hline Gpy(out)/anti & 1294.3 & 578.1 & 440.8 & 1295.8 & 574.6 & 438.8 & +27 & 76 \\
Gpy(out)/syn & 1288.8 & 580.9 & 441.8 & 1300.1 & 574.0 & 438.8 & +35 & 75 \\
Gpy(up)/anti & 1276.5 & 580.1 & 441.3 & 1293.4 & 570.9 & 437.1 & +26 & 76 \\
Gpy(up)/syn & 1275.5 & 581.1 & 441.6 & 1303.1 & 568.3 & 436.2 & +34 & 75 \\
& & & & & & & & \\
Gph(out)/anti & 1164.4 & 663.9 & 445.0 & 1167.7 & 657.9 & 453.1 & +23 & 78 \\
Gph(out)/syn & 1165.0 & 662.1 & 454.8 & 1172.9 & 654.4 & 452.9 & +31 & 76 \\
Gph(up)/anti & 1184.0 & 640.5 & 454.2 & 1177.9 & 640.2 & 452.8 & +24 & 76 \\
Gph(up)/syn & 1179.3 & 644.3 & 455.4 & 1176.8 & 643.0 & 454.1 & +33 & 75 \\
& & & & & & & & \\
Anti(ph)/anti & 1178.3 & 562.1 & 394.3 & 1171.9 & 563.5 & 395.1 & +12 & 80 \\
Anti(ph)/syn & 1175.1 & 562.7 & 394.4 & 1177.7 & 561.3 & 394.7 & +20 & 80 \\
Anti(py)/anti & 1183.4 & 556.5 & 392.4 & 1180.7 & 557.3 & 393.0 & +13 & 80 \\
Anti(py)/syn & 1177.3 & 560.3 & 393.5 & 1179.9 & 558.8 & 393.7 & +21 & 80 \\
Anti(up)/anti & 1177.7 & 557.3 & 392.6 & 1175.9 & 558.0 & 393.4 & +13 & 80 \\
Anti(up)/syn & 1175.2 & 559.2 & 393.4 & 1178.9 & 557.5 & 393.8 & +21 & 79
\end{tabular}

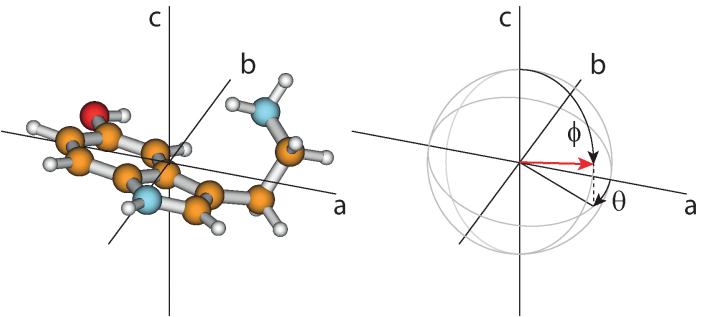

Fig. 2 On the left side the Gph(out)/anti conformer is shown in his principal axis system. The right side gives the definition of the angles $\theta$ and $\phi$ which describe the orientation of the TDM vector (red arrow) in non-planar molecules. $\theta$ is defined as the angle between the projection vector of the TDM in the $a b$-plane and the a-axis, and $\phi$ as the angle between the TDM and the $c$-axis.

Table 2 Comparison of the relative MP2/6-311++G(d,p) and CC2/ cc-pVTZ stabilities $\Delta E\left[\mathrm{~cm}^{-1}\right]$ in the electronic ground state of the most stable serotonin and tryptamine conformers. All calculations include zero-point energy corrections at the level of the optimization

\begin{tabular}{|c|c|c|c|c|c|}
\hline \multicolumn{2}{|c|}{ Tryptamine } & \multicolumn{4}{|l|}{$\underline{\text { Serotonin }}$} \\
\hline & & \multicolumn{2}{|c|}{$\underline{\mathrm{MP} 2 / 6-311++\mathrm{G}(\mathrm{d}, \mathrm{p})^{9}}$} & \multicolumn{2}{|c|}{ CC2/cc-pVTZ } \\
\hline \multicolumn{2}{|c|}{$\mathrm{MP} 2 / 6-311++\mathrm{G}(\mathrm{d}, \mathrm{p})^{43}$} & anti-5OH & syn-5OH & anti-5OH & syn-5OH \\
\hline Gpy(out) & 0.0 & 8.4 & 122.8 & 155.6 & 270.4 \\
\hline Gpy(up) & 125.6 & 93.7 & 243.4 & 158.1 & 285.1 \\
\hline Gph(out) & 136.4 & 0.0 & 375.3 & 0.0 & 330.5 \\
\hline Gph(up) & 304.3 & 352.9 & 482.3 & 252.9 & 351.5 \\
\hline Anti(py) & 482.3 & 524.3 & 629.6 & 710.4 & 790.8 \\
\hline $\operatorname{Anti}(\mathrm{ph})$ & 475.0 & 460.6 & 695.7 & 622.9 & 804.1 \\
\hline Anti(up) & 499.8 & 527.4 & 656.2 & 626.4 & 747.4 \\
\hline
\end{tabular}

For the anti-conformers $\theta$ is always smaller by $8^{\circ}$ than those of the respective syn counterparts.

In the case of tryptamine it has been shown that the most stable ground state conformer is Gpy(out) followed by Gpy(up) and Gph(out). ${ }^{40-42}$ Also for serotonin the Gpy(out)/anti conformer is the most stable ground state conformer according to density functional theory (DFT). ${ }^{5,9}$ Using functionals including dispersion interaction ${ }^{9}$ or changing to perturbation theory ${ }^{5,9}$ results in a change of the relative energy order, in agreement with the experimental findings from Cabezas et al. ${ }^{8}$ who found the $\mathrm{Gph}$ (out)/anti conformation to be the most stable one. The relative ground state energies of the seven most stable tryptamine conformers $^{43}$ are compared to those of the 14 serotonin conformers ${ }^{9}$ in Table 2 .

\subsection{High resolution electronic spectra of the serotonin conformers}

Fig. 3 shows the rotationally resolved spectrum of the electronic origin of the most intensive band in the laser induced fluorescence (LIF) spectrum recorded by LeGreve et al., ${ }^{5}$ denoted as serotonin $A$. It is accompanied by a simulation using the best parameters from a fit, given in Table 3 . The spectra and simulations of the other conformers $(B, C$, and $F)$ are given in the online ESI. $\dagger$ For all four conformers a similar redshift of around $13 \mathrm{~cm}^{-1}$ in the origin frequencies can be observed in comparison to the low-resolution values. ${ }^{5}$ The band type of all 


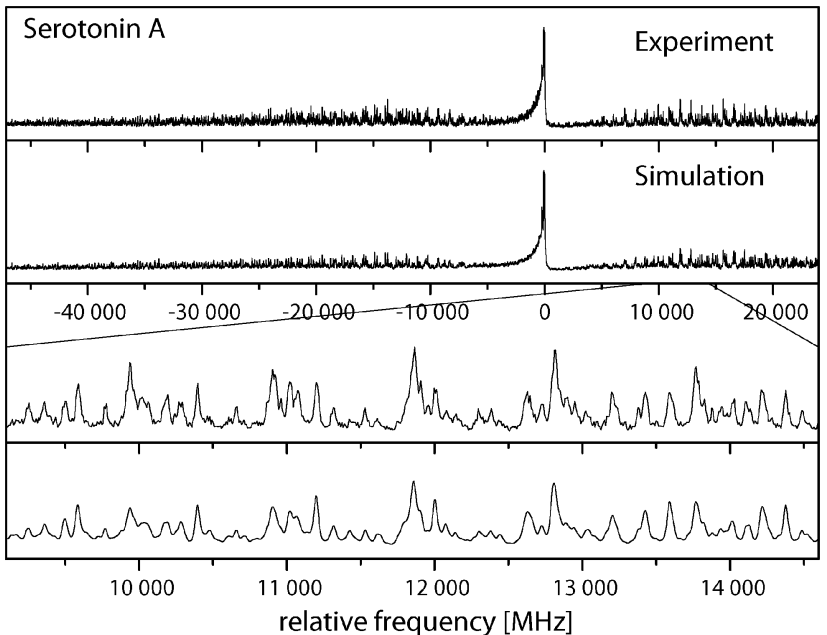

Fig. 3 Rovibronic spectrum of the electronic origin of the Gpy(out)/anti conformer.

conformers is mainly $a b$ hybrid as can be inferred from the values of $\theta$ and $\phi$ in Table 3. Excited state lifetimes range from 5 to $9 \mathrm{~ns}$ for conformers $A$ to $C$ and are larger than $12 \mathrm{~ns}$ for $F$.

The experimental parameters (rotational constants of the electronic ground $\left(A^{\prime \prime}, B^{\prime \prime}, C^{\prime \prime}\right)$ and excited state $\left(A^{\prime}, B^{\prime}, C^{\prime}\right)$, the inertial defect of both states $(\Delta I)$, the center frequencies of the respective bands $\left(\nu_{0}\right)$ and the orientation of the TDM) of the four conformers are given in Table 3 and are compared to the results of the $a b$ initio calculations. The assignments will be discussed in the next section.

\subsection{Conformational assignments}

For an assignment of the rovibronic bands to certain conformers we take a closer look at the rotational constants that are obtained from the analysis of the four spectra. Comparing the rotational constants of the $A, B$ and $F$ band with the calculated constants in Table 1, it is evident that they all belong to a gauche pyrrole conformation. For band $C$ an assignment to the gauche phenyl family can be made. However, within each family, the differences of the rotational constants are not large enough to make an unequivocal assignment.

LeGreve et al. ${ }^{5}$ assigned the group of transitions $F-H$ to the syn-conformers based on the origin shifts in 5-hydroxyindole (5OHI). If we follow this argumentation, bands $A$ and $B$ are members of the Gpy/anti family while band $F$ belongs to the $\mathrm{Gpy} /$ syn family. The shift of the electronic origins between band $A$ and $F$ amounts to $230.34 \mathrm{~cm}^{-1}$, which is very close to the shift of the respective syn- and anti-conformers in 5OHI $\left(230.91 \mathrm{~cm}^{-1}\right){ }^{12}$ Regarding the rotational constants, a difference of $+4.64 \mathrm{MHz}$ in the $A^{\prime \prime},-2.18 \mathrm{MHz}$ in the $B^{\prime \prime}$ and $-0.76 \mathrm{MHz}$ in the $C^{\prime \prime}$ constant is observed experimentally between band $A$ and $F$. For the anti- and syn-conformers of Gpy(out) (Gpy(up)) a change of $+5.5(+1.0) \mathrm{MHz}$ in the $A^{\prime \prime},-2.8(-1.0) \mathrm{MHZ}$ in the $B^{\prime \prime}$ and $-1.0(-0.3) \mathrm{MHz}$ in the $C^{\prime \prime}$ constant is calculated from the $a b$ initio optimized geometries. Hence, we assign the band $A$ to Gpy(out)/anti and the band $F$ to the respective syn-conformer. A confirmation of this assignment is given by the orientations of the transition dipole moments for the different conformers. It was mentioned that the CC2/cc-pVTZ calculations predict an increase of $8^{\circ}$ in the angle $\theta$ when we go from a anti to a syn-conformation. A shift by the same amount can be observed between the experimentally determined angles of serotonin $A$ and $F$. An additional affirmation can be extracted from the excited state lifetimes. In 5OHI it increases from 7.5 to $10 \mathrm{~ns}$ when going from the anti- to the synconformer. ${ }^{12}$ In the same manner the excited state lifetime increases from serotonin $A$ ( $6 \mathrm{~ns}$ ) to serotonin $F(>12 \mathrm{~ns})$.

For band $B$ we know that it belongs to the Gpy/anti family which has three members. Gpy(out)/anti is already assigned to band $A$ and Gpy(in)/anti is excluded by its high energy. Thus, the only possible assignment for band $B$ is to the Gpy(up)/anti conformer. This is supported by the differences between the experimental rotational constants of the $B$ conformer and the $a b$ initio calculated

Table 3 Experimentally determined molecular parameters for four different conformers $(A, B, C, F)$ of serotonin in their ground and first electronically excited states along with the ab initio calculated parameters of the respective conformers (Gpy(out)/anti, Gpy(up)/anti, Gph(out)/anti, Gpy(out)/syn). Changes of the rotational constants are defined as: $\Delta B_{g}=B_{g}^{\prime}-B_{g}^{\prime \prime}$, with $B_{g}$ as rotational constants with respect to the inertial axes $g=a, b, c$. Doubleprimed constants belong to the ground state and single-primed to the excited state. $\theta$ and $\phi$ define the angles of the TDM with the inertial axis system, $\nu_{0}$ is the center frequency of the respective band and $\tau$ the lifetime of the lowest electronically excited state

\begin{tabular}{|c|c|c|c|c|c|c|c|c|}
\hline & $A$ & Gpy(out)/anti & $B$ & Gpy(up)/anti & $C$ & Gph(out)/anti & $F$ & Gpy(out)/syn \\
\hline$A^{\prime \prime} / \mathrm{MHz}$ & $1286.50(1)$ & 1294.3 & $1267.28(1)$ & 1276.5 & $1163.12(1)$ & 1164.4 & $1281.86(1)$ & 1288.8 \\
\hline$B^{\prime \prime} / \mathrm{MHz}$ & $571.74(1)$ & 578.1 & $574.75(1)$ & 580.1 & $650.60(1)$ & 663.9 & $573.92(1)$ & 580.9 \\
\hline$C^{\prime \prime} / \mathrm{MHz}$ & 435.63(1) & 440.8 & 436.11(1) & 441.3 & $450.06(1)$ & 455.0 & 436.39(1) & 441.8 \\
\hline$\Delta I^{\prime \prime} / \mathrm{amu} \AA^{2}$ & -116.66 & -118.15 & -119.27 & -121.74 & -88.37 & -84.65 & -116.75 & -118.40 \\
\hline$A^{\prime} / \mathrm{MHz}$ & $1286.82(1)$ & 1295.8 & $1269.06(1)$ & 1293.4 & 1164.19(1) & 1167.7 & 1287.31(1) & 1300.1 \\
\hline$B^{\prime} / \mathrm{MHz}$ & $567.46(1)$ & 574.6 & $570.14(1)$ & 570.9 & $644.10(1)$ & 657.9 & $567.85(1)$ & 574.0 \\
\hline$C^{\prime} / \mathrm{MHz}$ & $433.20(1)$ & 438.8 & $433.89(1)$ & 437.1 & $447.13(1)$ & 453.1 & 433.59(1) & 438.8 \\
\hline$\Delta I^{\prime} / \mathrm{amu} \AA^{2}$ & -116.71 & -117.74 & -119.87 & -119.57 & -88.45 & -85.58 & -116.99 & -117.45 \\
\hline$\Delta A / \mathrm{MHz}$ & $+0.32(1)$ & +1.5 & $+1.78(1)$ & +16.9 & +1.07 & +3.3 & +5.45 & +11.3 \\
\hline$\Delta B / \mathrm{MHz}$ & $-4.28(1)$ & -3.5 & $-4.61(1)$ & -9.2 & -6.50 & -6.0 & -6.07 & -6.9 \\
\hline$\Delta C / \mathrm{MHz}$ & $-2.43(1)$ & -2.0 & $-2.22(1)$ & -4.2 & -2.93 & -1.9 & -2.80 & -3.0 \\
\hline$\theta /^{\circ}$ & \pm 38.83 & +27 & \pm 44.84 & +26 & \pm 34.60 & +23 & \pm 46.52 & +35 \\
\hline$\phi 1^{\circ}$ & 69.65 & 76 & 70.53 & 76 & 73.93 & 78 & 65.29 & 75 \\
\hline$\nu_{0} / \mathrm{cm}^{-1}$ & 32571.60 & 33029 & 32535.23 & 33007 & 32532.30 & 33023 & 32341.26 & 32653 \\
\hline$\tau / \mathrm{ns}$ & 6 & - & 5 & - & 9 & - & $>12$ & - \\
\hline
\end{tabular}


Table $4 \chi^{2}$ values $\left(\left(\left(A_{\text {exp }}^{\prime \prime}-B_{\text {exp }}^{\prime \prime}\right)-\left(A_{\text {calc }}^{\prime \prime}-B_{\text {calc }}^{\prime \prime}\right)\right)^{2} / \sigma_{A-B}^{2}+\left(\left(B_{\text {exp }}^{\prime \prime}-C_{\text {exp }}^{\prime \prime}\right)-\right.\right.$ $\left.\left.\left(B_{\text {calc }}^{\prime \prime}-C_{\text {calc }}^{\prime \prime}\right)\right)^{2} / \sigma_{B-C}^{2}+\left(\left(A_{\text {exp }}^{\prime \prime}-C_{\text {exp }}^{\prime \prime}\right)-\left(A_{\text {calc }}^{\prime \prime}-C_{\text {calc }}^{\prime \prime}\right)\right)^{2} / \sigma_{A-C}^{2}\right)$ obtained from experimental rotational constants $\left(A_{\text {exp }}^{\prime \prime}, B_{\text {exp }}^{\prime \prime}, C_{\text {exp }}^{\prime \prime}\right)$ and the standard deviations $\left(\sigma_{A}, \sigma_{B}, \sigma_{C}\right)$ of bands $A, B$, and $C$ taken from Table 3 . The calculated rotational constants ( $\left.A_{\text {calc, }}^{\prime \prime} B_{\text {calc, }}^{\prime \prime} C_{\text {calc }}^{\prime \prime}\right)$ for the conformers Gpy(out)/anti, Gpy(up)/anti, Gph(out)/anti and Gph(up)/anti are from Table 1

\begin{tabular}{lllll}
\hline Conformer & Gpy(out)/anti & Gpy(up)/anti & Gph(out)/anti & Gph(up)/anti \\
\hline$A$ & 504 & 29629 & 3561892 & 2327121 \\
$B$ & 52801 & 1541 & 2832778 & 1739439 \\
$C$ & 3258502 & 2625435 & 11406 & 71777
\end{tabular}

Gpy(up)/anti rotational constants $(\Delta A=+9.2 \mathrm{MHz}, \Delta B=$ $+5.3 \mathrm{MHz}, \Delta C=+5.2 \mathrm{MHz}$ ) which are very similar to the respective differences of the $A$ conformer $(\Delta A=+7.8 \mathrm{MHz}, \Delta B=$ $+6.4 \mathrm{MHz}, \Delta C=+5.2 \mathrm{MHz})$. A comparable deviation between theory and experiment can be expected for members of the same family. For the syn-conformers the deviations are higher, supporting the assignment that the grouping into syn- and anticonformers is correct.

In Table 4 the least squares of the differences of the rotational constants of the $A, B$, and $C$ conformers with the respective calculated values of anti-conformers of Gpy(out) and (up) and Gph(out) and (up) are determined. The $A$ band shows the lowest $\chi^{2}$ value and thus the best agreement with Gpy(out)/anti. The $B$ band has its best least square with Gpy(up)/anti and the $C$ band shows the best agreement with Gph(out)/anti.

An independent argument for this assignment arises from the comparison of the experimental ground state rotational constants in Table 3 with those from microwave spectroscopy. ${ }^{8}$ Cabezas et al. identified three conformers on the basis of the nuclear quadrupole constants as Gpy(out)/anti, Gpy(up)/anti and $\mathrm{Gph}$ (out)/anti. Since the rotational constants show an excellent agreement with our ground state values, the aforementioned assignment is confirmed unambiguously.

\subsection{Energetic differences and comparison to tryptamine}

Fig. 4 compares the relative energies of the 14 most stable conformers of serotonin, derived from CC2/cc-pVTZ calculations. It is evident that the $\operatorname{anti}(\mathrm{OH})$-conformers are always more stable in the electronic ground state and less stable in the electronically excited state. Furthermore, the energy difference between the syn- and anti-conformers is comparable within each electronic state if we exclude both Gph(out) conformers. In the electronic ground state the mean energetic difference amounts to $121 \pm 31 \mathrm{~cm}^{-1}$ and the corresponding value for the excited state is $264 \pm 43 \mathrm{~cm}^{-1}$. The values are comparable to those of 5 -hydroxyindole indicating that the energetic difference in both states is mostly unaffected by the addition of an ethyl amino side chain. ${ }^{12}$ For the Gph(out) conformers, however, the energetic gap in the $S_{0}$ state increases to $330 \mathrm{~cm}^{-1}$ while it is reduced to $81 \mathrm{~cm}^{-1}$ in the $S_{1}$ state. Thus, the conformation of the $\mathrm{OH}$ group either (i) stabilizes the anti-conformer in both electronic states by $200 \mathrm{~cm}^{-1}$ with respect to the syn-conformer or (ii) destabilizes the syn-conformer in both states by the same energy difference or (iii) does a mixture of both.

In order to answer this question we compare the conformational spaces of serotonin and tryptamine on the same level of theory, as shown in Fig. 5. The respective relative energies are given in Table 2. Most of the conformers follow a common trend. The relative energy of a conformer is comparable in tryptamine and the respective anti $(\mathrm{OH})$-conformation. Rotating the $\mathrm{OH}$-bond to the syn-conformation increases the relative energy by around $120 \mathrm{~cm}^{-1}$. Two conformers do not follow this trend: Anti(ph) and $\mathrm{Gph}$ (out). Judging from the distance between the $\mathrm{NH}_{2}$ group and the chromophore this points to a long-range effect between the $\mathrm{OH}$ and the $\mathrm{NH}_{2}$ group for Anti(ph). However, the most pronounced effect is observed for Gph(out) where the anti-conformation is stabilized significantly while the syn-conformer is destabilized by a comparable energy.

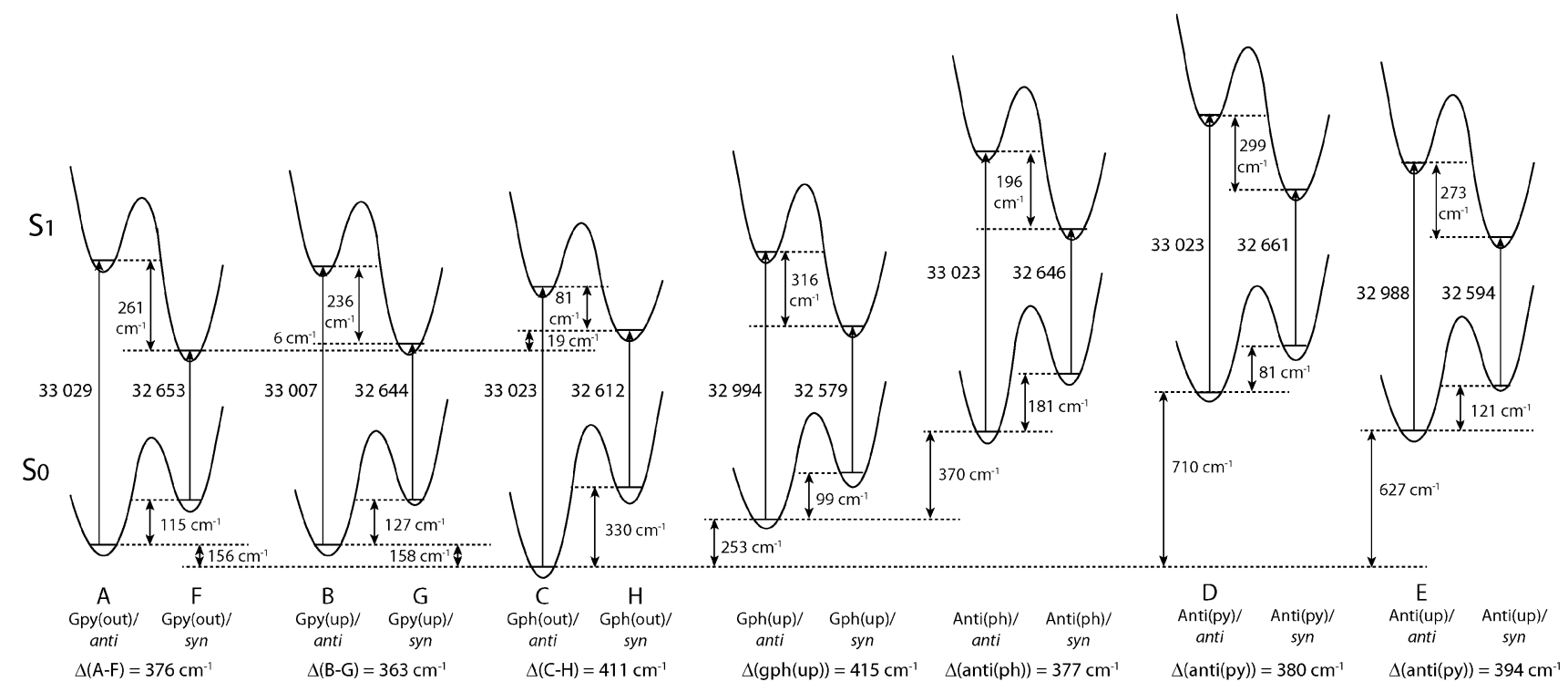

Fig. 4 Relative energies of the 14 most stable conformers of serotonin according to the CC2/cc-pVTZ calculations. All energies are zero-point corrected and given in $\mathrm{cm}^{-1}$. The letters refer to the designation of LeGreve et al. ${ }^{5}$ 


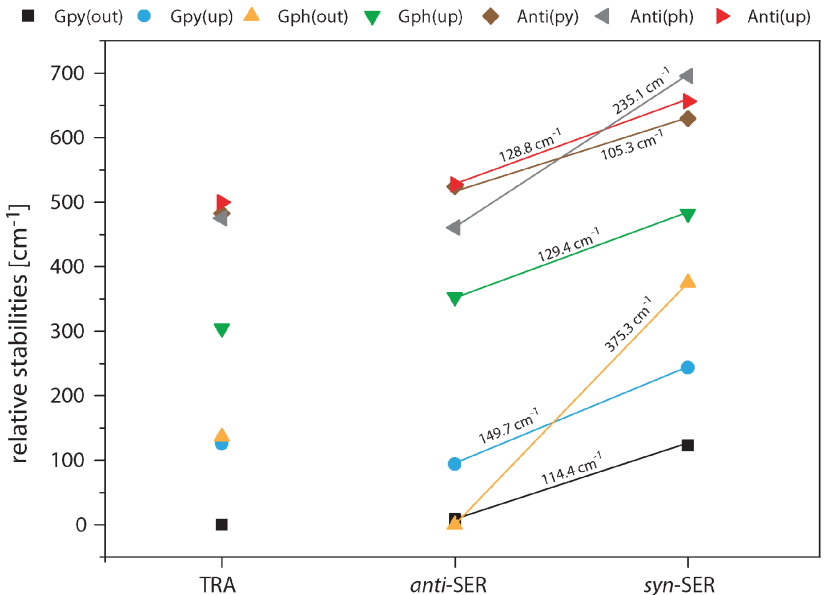

Fig. 5 Overview of the relative MP2/6-311++G(d,p) stabilities in the electronic ground state of the most stable serotonin (SER) and tryptamine (TRA) conformers. All calculations include zero-point energy corrections at the level of the optimization. Additionally, the energetic difference between the anti- and syn-conformers of serotonin are given.

In Fig. 6 we take a detailed look at the structures of the Gpy and Gph conformers of tryptamine and serotonin. In tryptamine

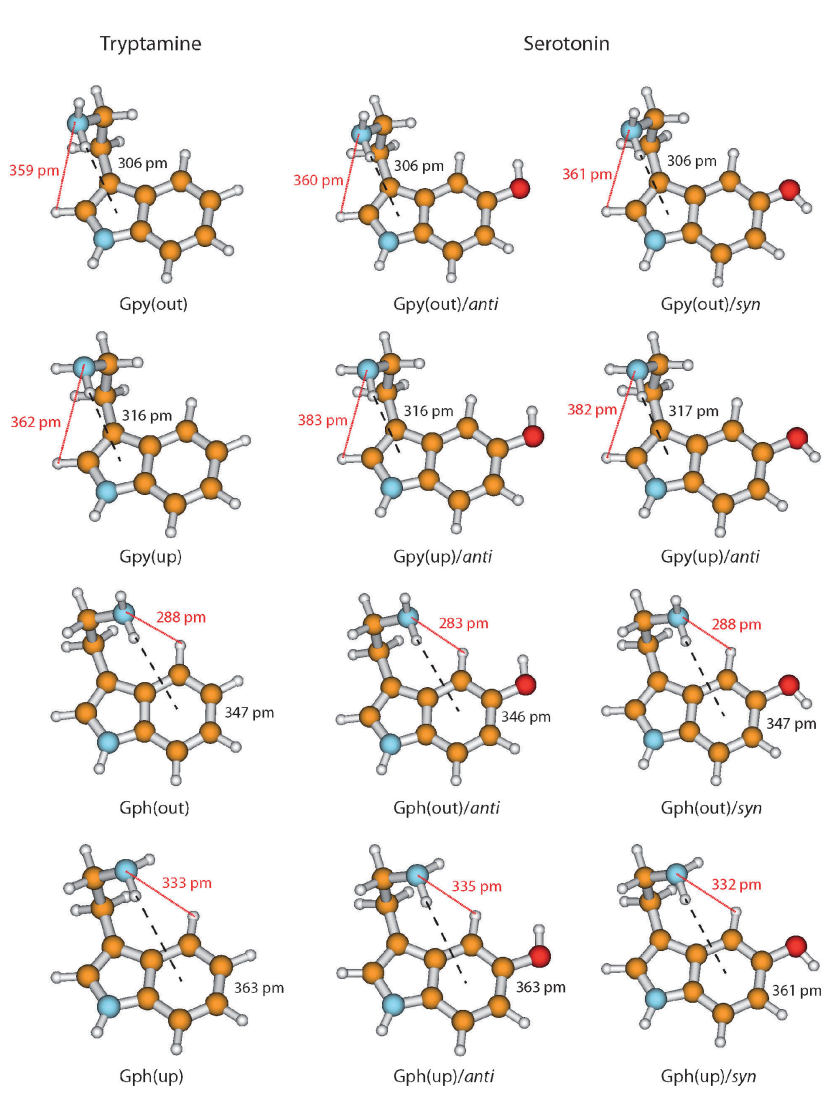

Fig. 6 Overview of the ground state structures of the Gpy and Gph conformers in tryptamine and serotonin. The structures are based on MP2/6-311++G(d,p) calculations. The distances between the amino hydrogen atom and the $\pi$ system at the pyrrole (Gpy) or phenyl (Gph) side of the indole chromophore (black dashed line) and the distances between the amino nitrogen atom and the closest hydrogen atom at the indole chromophore (red dotted line) are given in pm. the energetic ordering can be explained by the $\pi-\mathrm{H}$ distances which are increasing in the same way as the conformers are shifting towards higher energies. This confirms the explanation of a hydrogen bond formation between the amino hydrogen and the pyrrole (Gpy) or phenyl (Gph) $\pi$ cloud as given by Carney et $a .^{14}$ Addition of the hydroxyl group changes these distances only slightly, so that another effect must be responsible for the modulation of the excitation energy of the Gph(out) conformers.

A possible candidate for the additional stabilization is the interaction of the amino LP and an adjacent hydrogen atom at the indole chromophore ( $\mathrm{H} 2$ at $\mathrm{C} 2$ for the Gpy conformers and $\mathrm{H} 4$ at $\mathrm{C} 4$ for the Gph conformers, $c f$. Fig. 1). For the Gpy(up) and Gph(up) conformers of serotonin this can be excluded since the amino LP points away from the hydrogen atom. Also for Gpy(out) no effect is expected, because the distances do not change in comparison to tryptamine. For Gph(out)/anti, however, the amino LP is oriented towards the hydrogen atom H4 and the distance shortens from $288 \mathrm{pm}$ to $283 \mathrm{pm}$ by the addition of an hydroxyl group in anti orientation. In the case of the respective syn-conformer the LP of both the oxygen and the nitrogen atom are oriented towards $\mathrm{H} 4$. Hence, a competition of these LP to form a hydrogen bond or an electrostatic repulsion of both lone pairs seems to be a likely explanation for the destabilization of the syn-conformer and an increase of the amino LP hydrogen distance.

In this context one might ask why the additional stabilization through a hydrogen bond between the amino LP and H4 does not happen for the Gph(out) conformer in tryptamine. The answer for this can be given based on the natural charges in the indole chromophore as shown in Fig. 7.

In the pyrrole ring no or small changes between the natural charges of the tryptamine and serotonin conformers can be observed. This is different for the phenyl ring. Here, a pronounced increase in the electron density at C4 and C6 occurs, while C7 and especially C5 become more positive. The main difference between the syn- and anti-conformers of serotonin is the charge distribution at the carbon atoms $\mathrm{C} 4$ and C6: the electron density depends on the orientation of the lone pair and is either -0.24 or -0.27 . When the lone pair is pointing towards the respective atom, the negative charge is decreased. This trend is in agreement with the results from Srivastava and Singh ${ }^{9}$ and Oeltermann et al. ${ }^{12}$

We propose that the increase of electron density at the $\mathrm{C} 4$ atom increases the polarity of the $\mathrm{CH}$-bond which activates it for hydrogen bond formation. In tryptamine this activation is not possible. In turn, we assign the leading contribution of the stabilization of the Gph(out)/anti conformer to an intramolecular hydrogen-bond between the amino nitrogen LP and H4-atom at the phenyl ring. In general, a red shift of the $\mathrm{C} 4-\mathrm{H} 4$ stretch frequency due to a hyper-conjugative interaction between the lone pair of the nitrogen and the anti-bonding $\mathrm{CH}$ orbital is expected. The calculations, however, predict a blueshift of around $12 \mathrm{~cm}^{-1}$ between the $\mathrm{CH}$ vibrations of $\mathrm{Gph}$ (out)/anti and the other anti-conformers with Gpy, Gph and Anti conformation. ${ }^{9}$ It can be explained by the concept of "improper" hydrogen bonds. ${ }^{44}$ Following the argumentation of Joseph and 


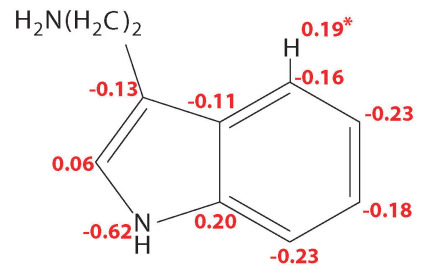

Tryptamine

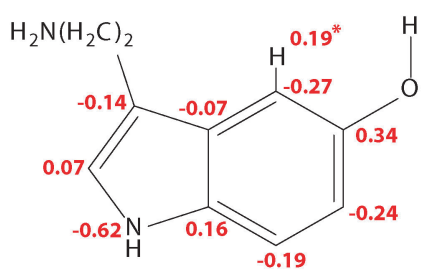

anti-Serotonin

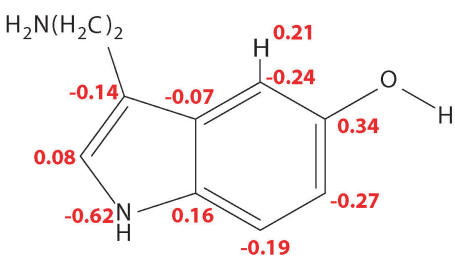

syn-Serotonin

Fig. 7 Natural charges from a Natural Population Analysis (NPA) for the Gph and Gpy conformers of tryptamine and serotonin in the ground state using the MP2/6-311++G(d,p) wave functions. * For the Gph(out) conformers of tryptamine and anti-serotonin the natural charge at $\mathrm{H} 4$ has a slightly higher value of 0.21

Jemmis ${ }^{45}$ hydrogen bonds can be decomposed into long-range and short-range interactions. In the former, the electron density of hydrogen bond acceptor leads to a polarization of the covalent bond. The resulting increase in the electrostatic interaction between $\mathrm{C}$ and $\mathrm{H}$ leads to a shortened bond length and in a blueshift of the stretch vibration. When the acceptor comes closer the hyperconjugative interaction leads in most cases to the familiar redshift. Mo et al. have computed the change in $\mathrm{CH}$ bond length for the system $\mathrm{F}_{3} \mathrm{C}-\mathrm{H} \cdots \mathrm{NH}_{3}$ which may provide a guideline for the discussed interaction. ${ }^{46}$ They found that for donor-acceptor distances larger than 210-240 pm the $\mathrm{CH}$ bond length is decreased. As in the present case the distance for the hydrogen bond is around $280 \mathrm{pm}$ it is likely that the electrostatic interaction is dominating, leading to the predicted blueshift.

In case of the Gph(out)/syn-conformer we postulate a destabilization mechanism which arises from an intramolecular competition between the amino nitrogen and oxygen lone pair for the hydrogen bond with the H4-atom. This is backed by the computed C4-H4 stretch frequencies for the Gph(out)/syn and the other syn-conformers with Gpy, Gph and Anti conformation which show a similar blue shift of around $9 \mathrm{~cm}^{-1},{ }^{9}$ close to our MP2 values.

A closely related system is mexamine (5-methoxytryptamine). The exchange of the hydroxyl group by an methoxy group leads to a decrease in the total number of observed conformers. ${ }^{20}$ Like in serotonin, also for 5-methoxytryptamine theoretical calculations predict the $\mathrm{Gph}(\mathrm{out}) /$ anti as lowest energy conformer in the ground state. Additionally, the calculated energy gap between syn and anti increases for the Gph(out) by $200 \mathrm{~cm}^{-1}$ which is in good agreement with the values presented here for serotonin. Hence, the same effect is expected to be responsible for the shape of the mexamine conformational space as well. The same trend was observed for 5-methoxy $\mathrm{N}$-acetyl tryptophan methyl amide, where only one conformer is observed in the IR-UV holeburn spectrum. ${ }^{22}$ This is assigned to a structure in which one of the LP of an oxygen atom is pointing towards $\mathrm{H} 4$. Also for the 5-methoxy substituted $N$-acetyltryptamine (melatonin) ${ }^{21}$ the anti-conformers are selectively stabilized. For the two most abundant conformers a hydrogen bond is formed between $\mathrm{H} 4$ and the $N$-acetylamino side chain: either with the LP of the oxygen or the nitrogen atom. Thus, the stabilization of anticonformers via oxygen atoms connected to position 5 in the indole chromophore seems to be a general effect in these class of neurotransmitters.

\section{Conclusions}

Four conformers of serotonin were analyzed and assigned to the Gpy(out)/anti (band $A$ ), Gpy(up)/anti (band $B$ ), Gph(out)/ant $i$ (band $C$ ) and Gpy(out)/syn (band $F$ ) structures on the basis of the rotational constants and other molecular parameters from rotationally resolved electronic spectra. The orientation of the hydroxyl group is deduced from a characteristic difference in the TDM orientation by $8^{\circ}$. Moreover, the excited state lifetime is an additional molecular property that shows significant changes depending on the hydroxyl group orientation. Our results confirm the assignment made by LeGreve et al. based on vibrationally resolved spectroscopy. ${ }^{5}$ The addition of a hydroxyl group at position 5 to tryptamine in $\mathrm{Gph}$ (out) confirmation leads to a stabilization of the respective anti $(\mathrm{OH})$ - and destabilization of the syn-conformer in the ground state. As a consequence $\mathrm{Gph}(\mathrm{out}) /$ anti becomes the most stable conformer of serotonin, while for tryptamine this is Gpy(out). The energetic ordering in tryptamine is based on the $\mathrm{H}-\pi$ interaction between the amino hydrogen and the indole chromophore. For the Gph(out) conformers of serotonin an additional hydrogen bond between the LP of the amino group and the $\mathrm{H} 4$ atom at the indole chromophore is formed. This additional hydrogen bond selectively stabilizes the respective anticonformer. For the syn-conformer a destabilization occurs due to a intramolecular competition between the $\mathrm{O} \cdots \mathrm{H} 4$ and $\mathrm{N} \cdots \mathrm{H} 4$ hydrogen bonds.

Since the anti-conformers are always more stable in the ground state and the syn-conformers in the excited state ( $c f$. Fig. 4) a stabilization of $\mathrm{Gph}(\mathrm{out}) /$ anti and a destabilization of $\mathrm{Gph}$ (out)/syn leads to an increase of the syn-anti gap in the ground state and a decrease in the excited state. This might be the reason for the larger experimental difference in the excitation energies between both Gph(out) conformers.

The present results illustrate that even substituents which do not interact directly can communicate via competitive interactions and have a large influence on the shape of the conformational space. We could show that for various 5-substituted tryptamines (5-hydroxytryptamine, 5-methoxytryptamine, $N$-acetyl-5-methoxytryptamine, 5-methoxy $N$-acetyl tryptophan methyl amide) the hydrogen bond between the flexible side chain and $\mathrm{H} 4$ is a central motif which governs the most stable structure that can be experimentally observed. 


\section{Acknowledgements}

Financial support of the Deutsche Forschungsgemeinschaft via grant SCHM1043 12-1 is gratefully acknowledged. Christian Brand acknowledges financial support from the Alexander von Humboldt foundation through a Feodor-Lynen fellowship. We thank the Universitätsrechenzentrum Köln for granted computing time and Olivia Oeltermann for assistance with the experiments.

\section{References}

1 D. E. Nichols and C. D. Nichols, Chem. Rev., 2008, 106, 1614-1641.

2 D. Hoyer, D. E. Clarke, J. R. Fozard, P. R. Hartig, G. R. Martin, E. J. Mylecharane, P. R. Saxena and P. P. A. Humphrey, Pharmacol. Rev., 1994, 46, 157-203.

3 F. Boess and I. Martin, Neuropharmacology, 1994, 33, 275-317.

4 M. Berger, J. A. Gray and B. L. Roth, Annu. Rev. Med., 2009, 102, 355-366.

5 T. A. LeGreve, E. E. Baquero and T. S. Zwier, J. Am. Chem. Soc., 2007, 129, 4028-4038.

6 A. Lagutschenkov, J. Langer, G. Berden, J. Oomens and O. Dopfer, J. Phys. Chem. A, 2010, 114, 13268-13276.

7 T. A. LeGreve, W. H. James III and T. S. Zwier, J. Phys. Chem. A, 2009, 113, 399-410.

8 C. Cabezas, M. Varela, I. Peña, J. C. López and J. L. Alonso, Phys. Chem. Chem. Phys., 2012, 14, 13618-13623.

9 S. K. Srivastava and V. B. Singh, RSC Adv., 2015, 5, $28141-28157$.

10 T. van Mourik and L. E. V. Emson, Phys. Chem. Chem. Phys., 2002, 4, 5863-5871.

11 S. Arnold and M. Sulkes, Chem. Phys. Lett., 1992, 200, 125-129.

12 O. Oeltermann, C. Brand, M. Wilke and M. Schmitt, J. Phys. Chem. A, 2012, 116, 7873-7879.

13 W. Caminati, Phys. Chem. Chem. Phys., 2004, 6, 2806-2809. 14 J. R. Carney and T. S. Zwier, J. Phys. Chem. A, 2000, 104, 8677. 15 L. A. Philips and D. H. Levy, J. Chem. Phys., 1988, 89, 85-90. 16 Y. R. Wu and D. H. Levy, J. Chem. Phys., 1989, 91, 5278-5284.

17 T. Nguyen, T. Korter and D. Pratt, Mol. Phys., 2005, 103, 1603-1613.

18 M. Schmitt, M. Böhm, C. Ratzer, C. Vu, I. Kalkman and W. L. Meerts, J. Am. Chem. Soc., 2005, 127, 10356-10364.

19 M. Böhm, R. Brause, C. Jacoby and M. Schmitt, J. Phys. Chem. A, 2009, 113, 448-455.

20 T. B. C. Vu, I. Kalkman, W. L. Meerts, C. Brand, Y. N. Svartsov, S. Wiedemann, R. Weinkauf and M. Schmitt, Phys. Chem. Chem. Phys., 2009, 11, 2433-2440.

21 J. T. Yi, C. Brand, M. Wollenhaupt, D. W. Pratt, W. L. Meerts and M. Schmitt, J. Mol. Spectrosc., 2011, 268, 115-122.

22 B. C. Dian, G. M. Florio, J. R. Clarkson, A. Longarte and T. S. Zwier, J. Chem. Phys., 2004, 120, 9033-9046.

23 M. Schmitt, J. Küpper, D. Spangenberg and A. Westphal, Chem. Phys., 2000, 254, 349-361.

24 S. Gerstenkorn and P. Luc, Atlas du spectre d'absorption de la molécule d'iode 14800-20000 $\mathrm{cm}^{-1}$, CNRS, Paris, 1986.

25 R. Ahlrichs, M. Bär, M. Häser, H. Horn and C. Kölmel, Chem. Phys. Lett., 1989, 162, 165-169.

26 J. T. H. Dunning, J. Chem. Phys., 1989, 90, 1007-1023.
27 C. Hättig and F. Weigend, J. Chem. Phys., 2000, 113, 5154-5161.

28 C. Hättig and A. Köhn, J. Chem. Phys., 2002, 117, 6939-6951.

29 C. Hättig, J. Chem. Phys., 2002, 118, 7751-7761.

30 P. Deglmann, F. Furche and R. Ahlrichs, Chem. Phys. Lett., 2002, 362, 511-518.

31 TURBOMOLE V6.5 2013, a development of University of Karlsruhe and Forschungszentrum Karlsruhe $\mathrm{GmbH}$, 1989-2007, TURBOMOLE GmbH, since 2007; available from http://www.turbomole.com.

32 M. J. Frisch, G. W. Trucks, H. B. Schlegel, G. E. Scuseria, M. A. Robb, J. R. Cheeseman, G. Scalmani, V. Barone, B. Mennucci, G. A. Petersson, H. Nakatsuji, M. Caricato, X. Li, H. P. Hratchian, A. F. Izmaylov, J. Bloino, G. Zheng, J. L. Sonnenberg, M. Hada, M. Ehara, K. Toyota, R. Fukuda, J. Hasegawa, M. Ishida, T. Nakajima, Y. Honda, O. Kitao, H. Nakai, T. Vreven, J. A. Montgomery, Jr., J. E. Peralta, F. Ogliaro, M. Bearpark, J. J. Heyd, E. Brothers, K. N. Kudin, V. N. Staroverov, R. Kobayashi, J. Normand, K. Raghavachari, A. Rendell, J. C. Burant, S. S. Iyengar, J. Tomasi, M. Cossi, N. Rega, J. M. Millam, M. Klene, J. E. Knox, J. B. Cross, V. Bakken, C. Adamo, J. Jaramillo, R. Gomperts, R. E. Stratmann, O. Yazyev, A. J. Austin, R. Cammi, C. Pomelli, J. W. Ochterski, R. L. Martin, K. Morokuma, V. G. Zakrzewski, G. A. Voth, P. Salvador, J. J. Dannenberg, S. Dapprich, A. D. Daniels, Ö. Farkas, J. B. Foresman, J. V. Ortiz, J. Cioslowski and D. J. Fox, Gaussian 09 Revision E.01, Gaussian Inc, Wallingford CT, 2009.

33 A. E. Reed, R. B. Weinstock and F. Weinhold, J. Chem. Phys., 1985, 83, 735-746.

34 A. Ostermeier, A. Gawelczyk and N. Hansen, in Step-Size Adaptation Based on Non-Local Use of Selection Information, ed. Y. Davidor, H.-P. Schwefel and R. Männer, Springer, 1994, pp. 189-198.

35 N. Hansen and A. Ostermeier, Evolutionary Computation, 2001, 9, 159-195.

36 W. L. Meerts, M. Schmitt and G. Groenenboom, Can. J. Chem., 2004, 82, 804-819.

37 W. L. Meerts and M. Schmitt, Phys. Scr., 2005, 73, C47-C52.

38 W. L. Meerts and M. Schmitt, Int. Rev. Phys. Chem., 2006, 25, 353-406.

39 M. Schmitt and W. L. Meerts, in Handbook of High Resolution Spectroscopy, ed. M. Quack and F. Merkt, John Wiley and Sons, 2011.

40 Y. K. Sturdy and D. C. Clary, Phys. Chem. Chem. Phys., 2007, 9, 2065-2074.

41 M. Schmitt, R. Brause, C. Marian, S. Salzmann and W. L. Meerts, J. Chem. Phys., 2006, 125, 124309.

42 J. R. Clarkson, B. C. Dian, L. Moriggi, A. DeFusco, V. McCarthy, K. D. Jordan and T. S. Zwier, J. Chem. Phys., 2005, 122, 214311.

43 N. Mayorkas, A. Bernat, S. Izbitski and I. Bar, J. Chem. Phys., 2013, 138, 124312.

44 P. Hobza, V. Špirko, H. L. Selzle and E. W. Schlag, J. Phys. Chem. A, 1998, 102, 2501-2504.

45 J. Joseph and E. D. Jemmis, J. Am. Chem. Soc., 2007, 129, 4620-4632.

46 Y. Mo, C. Wang, L. Guan, B. Braïda, P. C. Hiberty and W. Wu, Chem. - Eur. J., 2014, 20, 8444-8452. 